\title{
Cockroach 0.0 Beta: Exploring the novel building performance simulation domain of pest modelling through a literature review
}

\author{
Clarice Bleil de Souza ${ }^{1}$, Pieter de Wilde ${ }^{2}$ \\ ${ }^{1}$ Welsh School of Architecture, Cardiff University, Cardiff, Wales - UK \\ King Edward VII Avenue, CF10 3NB bleildesouzac@cardiff.ac.uk \\ ${ }^{2}$ Chair of Building Performance Analysis, School of Art, Design and Architecture, Plymouth \\ University, Plymouth - UK, Roland Levinsky Building, Drake Circus, PL4 8AA
}

\begin{abstract}
This paper explores the novel building performance simulation domain of pest modelling. Despite extensive knowledge on pest management in agriculture, pest behaviour and their impact in epidemiology, building science currently has no computational formulas, algorithms or simulation tools to quantify the vulnerability of the built environment in relation to pests. Therefore, it is not possible for planners and the many other different stakeholders involved in the construction industry to project scenarios to study future operation of buildings in relation to pest prevention and control. A comprehensive literature review is presented to provide a high level outline of this new area in building performance simulation. Findings are used to discuss transferability of different types of pest models and approaches from other areas to the built environment, pointing out avenues for future research.
\end{abstract}

\section{Introduction and background}

Ecological imbalance, climate change, rapid urbanization and growth have been responsible for an increase in the number of pest borne diseases and pest damage to buildings. Pests are a major problem for humans. They pose a risk to the health of building occupants via the spread of bacteria, viruses and parasites; they cause damage to building structures, fabric and content, and increase fire risks by gnawing electricity cables. However, despite being a recognized public health problem by the World Health Organisation (WHO) and many governments, pest control remains mainly in the hands of private companies which benefit from a market of around \$18bn (Rentokil 2015). These companies tend to approach the pest problem using strategies of 'building inspection - advice - treatment (mostly chemical) building maintenance program' whereas pests are actually a systemic problem which also needs to be dealt at the level of 'legal action, education, institutional capacity, building and research at international, national and local levels' (Bonnefoy et al., 2008).

The literature provides a comprehensive list of what attracts pests to the built environment. It also shows a comprehensive amount of information related to pest control, whereas pest modelling and the presence of pests are mainly documented in the domains of biogeography, epidemiology and agriculture. However, so far these models and approaches have not been taken up within the domain of building science: pest modelling is not an active area that attracts efforts within the building performance simulation community. Thus there are no tools to project scenarios for planners and different stakeholders in the construction industry to design and operate buildings in relation to pest infestation prevention and pest control. As a result, architects engineers and other key actors in building design lack access to a comprehensive approach to design pests out of buildings.

The aim of this paper is to provide an initial high level outline of a new area in building performance simulation: The simulation of pest vulnerability of the built environment. We propose to reach this aim through a comprehensive literature review, presenting and discussing current models for simulating pests in the outdoor environment (at a descriptive level and to quantify results of interventions), followed by methods to gauge the effect of interventions undertaken to control pests inside buildings, finishing with a set of guidelines to pest proof buildings at the level of building envelope and immediate surroundings (Figure 1).

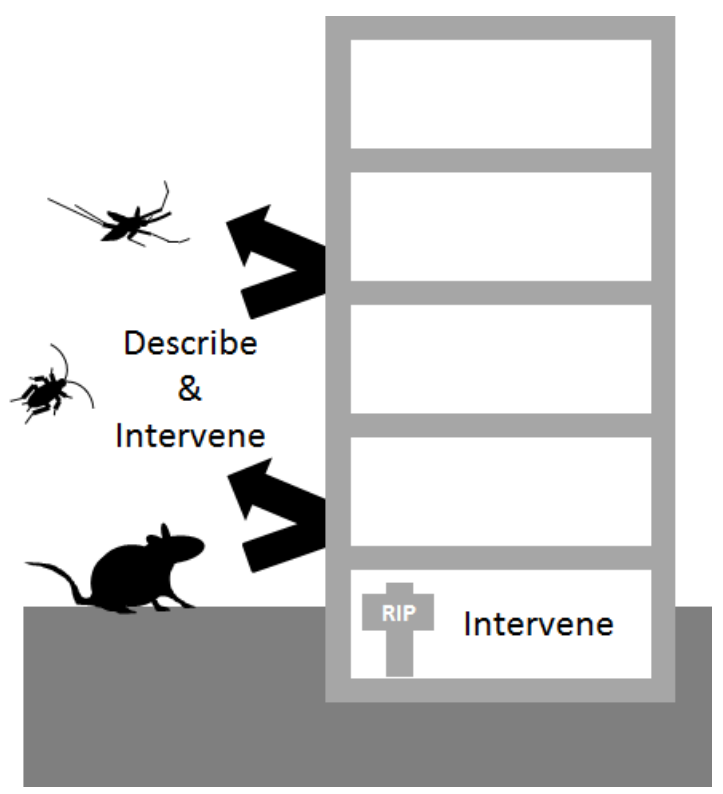

Figure 1: Current types of pests simulation models 


\section{Methodology}

The authors undertook a systematic review of the literature related to pest modelling followed by a qualitative Thematic Analysis on the content of these articles. Thematic Analysis is a 'qualitative datareduction and sense-making effort that takes a volume of qualitative material and attempts to identify core consistencies and meanings' (Patton 2002). This type of analysis enabled the construction of a classification system to order the different modelling approaches in terms of what is transferrable to the built environment according to the following set of criteria:

- Environment being modelled

- Data used to produce the model;

- $\quad$ Purpose of the modelling.

There are mainly two types of environment being modelled when pests are involved: 'Outdoor environments', with modelling resolution varying from regional to neighbourhood scale and 'Indoor environments', which focus on pest populations inside buildings. Outdoor models are mainly spatial and are normally implemented via GIS.

Models can be mainly divided into those which deal with insects and those which deal with mammals, but the type of species being modelled varies significantly. However, the data used to produce these models tends to mainly come from traps, normally used to detect the presence or absence of pests, in combination with a series of environmental and anthropogenic features such as climate, vegetation/crop, urban characteristics, socioeconomic level, sanitation, etc. Variations in combining these different types of data and data sources depend on the purpose and scale of the modelling as well as the type of species being modelled.

The purpose of modelling varies depending on different disciplines. Models from biogeography and conservation of bio-diversity tend to be descriptive, and are designed to quantify pest distribution, size of population, severity of infestation, etc., whereas models from biosecurity and agriculture tend to be used for risk assessment, monitoring, control, gauging future incidence, etc. Models from epidemiology and public health normally focus on connecting the incidence of different illnesses directly to pest population decay and are used mainly to control and monitor different types of interventions, from the use of chemicals to sanitation.

The following sections explores pest modelling in relation to the aforementioned themes preparing a discussion in terms of what is transferable to the built environment.

\section{Descriptive models of the outdoor environment}

Since the early 90's, GIS has been used to 'produce maps that predict the probability of detecting species over a given area' (Sacchi et al., 2008) without the need to undertake direct surveys to be used in planning pest control operations and surveillance. The possibilities of combining different types of information (all geographically referenced) and data types of different nature including variations throughout time, enables more sophisticated probabilistic models to be developed based on geostatistics.

Classically defined as 'the study of spatially continuous processes using partially discrete observations at a finite number of locations' (Diggle et al., 2013), geostatistical models address spatio-temporal data sets and are normally focused on predictions. They are widely used in epidemiology and agriculture and comprehend a series of different methods or algorithms. Spatio-temporal distributions can be linked with other parameters which affect or explain pest behaviour and therefore may be useful to inform prevention, enhancing the effectiveness of pest control strategies. Information to produce these maps tend to come from spacialization of data collected from multiple traps, sometimes in combination with thematic maps, landscape and climate based models.

\section{Models based on data coming from traps}

There are different discussions as to what is the best way to distribute traps: randomly, in a grid, in specific habitats, etc. and also different discussions and algorithms to extrapolate data from traps and spatially distribute them.

Petrovskii et al. (2014) explore the extrapolation of population density from single traps based on the use of Brownian motion and Levy flights models to investigate random walks for insect movement around a trap. This method is limited to extrapolating population densities to only the vicinity of the trap.

The FAO/IAEA Programme (2006) proposes the use of Inverse Distance Weighted (IDW) models, spline and Kriging as suitable interpolation methods for pest distribution in agriculture when traps are evenly distributed in a single grid. These algorithms provide reasonably accurate results if the population distribution is homogenous.

Petrovskii et al. (2014) explore the projection of pest density population over a certain area by proposing that the interpolation of data collected from several traps can be done via numerical integration using whatever method provides the fastest convergence rate, when the distribution of the population is simple. However, if the population distribution is patchy and with complicated spatial patterns, the accuracy of the integration will heavily depend on the location of nodes, in the grid where traps are installed, in relation to the position of the patches. As these patches are for the vast majority unknown, the authors propose that population density could be estimated based on a "probabilistic integration across a group of fields or habitats with similar properties', based on a small number of randomly distributed traps.

\section{Thematic maps}

Traweger and Slotta- Bachmayr (2005) present a study to assess the suitability of urban environments to support rats using a combination of landscape characteristics maps 
correlated with literature data on foraging and habitat, validating predictions through the use of randomly distributed traps. Sacchi et al. (2008) propose a GIS model of the distribution of rats based on the interpolation of data coming from non-poisonous baits, placed over suitable habitats in an urbanised area, in combination with thematic maps.

Tamayo-Uria et al. (2014) present an example of using the Log-Gaussian Cox Process to provide a description of rat sighting in an urban area of Madrid, coupled with a conditional intensity function which connects variation of sighting through time with potentially useful explanatory variables. They conclude that the intensity of sightings is inversely proportional to the age of the building and its distance to vegetated areas, markets and cat feeding stations and that sighting is also affected by seasonal variations.

\section{Landscape and climate models}

According to Petrovskii, et al., (2014), the 'environment is known to affect the population dynamics in space and time through a variety of specific factors such as landscape structure, seasonality and solar cycles, weather conditions on both global and regional scales, etc.' In order to understand if variations in the population come from landscape properties, weather conditions or are dependent on self-regulation mechanisms, data from traps tend to be combined with landscape, climate and other thematic features.

Sutherst (2014) provides a comprehensive literature review on different models considering the climate mainly focusing on those which take into account animal physiological responses to climate, climatic factors which affect the occurrence of species and correlations between pest populations and plant growth. He explains the development of CLIMEX which uses soil moisture to simulate a plant growth index which together with parameterised temperature and moisture response functions can be used to describe growth conditions for insects. CLIMEX has a temporal resolution of 1 week and works in different latitudes, climate zones, seasons and is suitable for tracking migration routes. Sutherst warns against species modelling based on trap data only and notes that input from experts who understand the requirements and behaviour of the species is crucial to guarantee valid results. The vast majority of landscape and climate based models are developed to assess pest management in agriculture and there are several types of these models, such as:

- Phenological models, which focus on how cyclic and seasonal natural phenomena affect pest life cycle (Caffarra et al. 2012).

- Physiologically based demographic models which are 'models for population dynamics based on physiological responses at individual level to environmental driving variables' (Gilioli et al.,
2016), i.e. development, fecundity and mortality rate functions.

- Combinations of crop growth models with pest life models which can be found in Shirley et al. (2001), Donatelli et al. (2017) to cite a few.

\section{Predictive models of interventions in the outdoor environment}

Pest control is normally assessed in terms of economic and ecologic losses pests may cause. Quantitatively, this means keeping pest population bellow certain thresholds, which in agriculture are defined based on the cost of taking action versus the costs involved in production losses. The two main indices used to judge when control actions should be undertaken are (Georgescu and Zhang, 2010):

- The Economic Injury Level (EIL) defined as the 'lowest pest density which causes economic damage', i.e. a minimum point at which pest population needs to be held under control, rather than reaching extinction.

- Economic threshold (ET) level, defined as the 'lowest pest density at which control measures should be taken so that the EIL is not exceeded'.

These thresholds can be achieved either using low environmental impact methods or high environmental impact methods. However, decisions related to management and control can become quite complicated when several pests and several crops are being considered, because a multitude of potential actions are available. As result, it is also possible to find literature on decision support system models to aid farmers and agricultural technicians to take effective actions (see for instance del Aguila et al., 2015). These models are basically expert systems sometimes with a certain degree of Artificial Intelligence within them.

\section{Models of low environmental impact}

Low environmental impact control methods are defined as the ones used to 'minimize the damage caused to nontarget organisms' (Georgescu and Zhang, 2010), parts of the ecosystem which are not the pest being monitored and controlled. These methods include mechanical interventions such as traps, barriers, etc. and biological methods such as using biological insecticides, predators, inserting sterile pest individuals to avoid pest breeding, spreading pest specific related diseases, etc. (ibid.).

In the literature, most models designed to gauge pest control interventions using low environmental impact methods are developed to be used in agriculture. Most of these models seem to be based on Impulsive differential equations as these are seen as appropriate mathematical representations to describe decline of pest population after an intervention happens; these are impulsive events such as the introduction of predators, diseases, etc. (Akman $e t$ al., 2015). According to Georgescu and Zhang (2010) there are normally two types of models: time dependent models, used when pest controls are implemented on a time-basis strategy; and state dependent models, used 
when controls are implemented every time the size of the population reaches the economic threshold. Georgescu and Zhang (2010) present a model designed to gauge interventions based on releasing, at a constant rate, ineffective pest individuals, contaminated with a specific pest disease, together with natural predators. An impulsively perturbed differential model is introduced with a prey dependant functional response, i.e. 'a functional response which depend on the size of the prey population' (ibid.). Wang et al. (2011) also use impulsive differential equations to examine the predator-prey interaction 'describing the interaction between the pest and natural enemy with impulsive and periodic enemy releases'. Akman et al. (2015) propose impulsive differential equations to accommodate fluctuations in pest birth rates based on environmental and climatic factors affecting egg production. Fluctuations in birth rates provide further refinements to models such as the one proposed by Georgescu and Zhang (2010) and can also be added to in models which simulate chemical interventions.

Murray et al. (2014) propose a Bayesian Network model to graphically represent the environment for rabbits' persistence integrating climatic, environmental (including habitat) and management variables. The model is used to produce GIS spatial risk maps considering how susceptible habitats are to be colonised and how suitable they are for rabbits to persist. The conditional probability model and dependencies between variables were assembled based on data collected from a workshop with experts. Since management variables were included, scenarios of different types of natural interventions can be assessed: the consequences of using barriers/fences in rabbit's distribution; the effectiveness of shooting, baiting and warren ripping in the ability of the rabbit's population to persist; as well the effects of different specific types of disease control and predators.

\section{Models of high environmental impact}

High environmental impact methods are defined as the ones using chemical pesticides to control or eradicate pest populations. The use of pesticides is seen as economically effective because 'the costs of chemical products and their application is usually lower than the economic losses caused by pests' (del Aguila et al., 2015).

Despite efforts on the exploration of new selective chemical pesticides and the application of chemicals considering specific stages of pest development, the persistent use of pesticides is still seen as a problematic control measure. It often leads to the emergence of pest resistant populations and there is no proof it is efficient to control pests under continuous or permanent states of migration (Stejskal 2002). Furthermore, the use of chemical pesticides is 'a major threat to beneficial insects, which are sometimes more affected by pesticide spraying than targeted pests' (Georgescu and Zhang, 2010). Therefore, it is not uncommon to find studies which focus on modelling the use of low environmental impact methods together with chemicals when reaching appropriate economic thresholds.
An important component of these models is that the frequency of natural enemy releases cannot coincide with that of the pesticide application, especially when pesticides also affect the population of predators. Tang et al. (2011) explores in detail three different possibilities of combinations of pesticide spray and predator release: spraying pesticide more often than releasing predators, releasing predators more often than spraying pesticides or releasing predators frequently, and using pesticides only when is the pest population still reaches the economic threshold. They model these possibilities while varying initial pest densities, releasing rate and amount of predators and pesticides, using impulse differential equations.

Mamedov and Udalov (2002) have developed CENOCON, a tool to run several models for the different agents involved in simulating an ecosystem. Agents are mainly plants and animals. Plants grow and animals move, eat and breed. Within this ecosystem, 'individuals interact with each other establishing and developing the community as a whole'. Organisms have individual and population specific parameters (e.g. they are herbivorous or carnivorous, etc.). The program generates a virtual space in which a grid of cells is set up with plant seeds randomly distributed throughout them. The ecosystem then follows a grow development path according to data provided for each agent. The model is used to investigate the optimal moment to introduce chemicals to specifically kill two types of pests minimising harmful effects on beneficial predators. Six agents were involved in the simulation and chemicals were applied at two different dates in relation to predator colonization (before and after).

Zhang and Swinton (2009) suggest combining pesticide spray with predator release and present a bio-economic study in which predator-prey interaction combined with crop growth and yield damage are evaluated in an optimization framework. The result of integrating biological with economic models produced a new threshold: 'the natural enemy-adjusted economic threshold (...) defined as the pest population density threshold at which insecticide control becomes optimal in spite of the opportunity cost of injury to natural enemies of the target pest'. A Lotka-Volterra (predator-prey) equation is used to describe predator population density changes over discrete time periods combined with a yield response function and a crop revenue objective function.

When looking at simulation with chemical interventions only, the literature presents a wide range of software developed to 'simulate volatile pesticide transport by including a description of the volatilization process at the soil-air interface' (Luo et al., 2012). However, little is known in relation to the effects of emission reduction strategies in the efficacy of pest control strategies. Luo et al. (2012) propose a simulation model, validated through experimental measurements, to assess several emission reduction strategies and their effect in pest reduction. First order decay reaction models are used to gauge fumigant fate by considering different degradation rates at the liquid, gas and solid states and a logistic dose response 
curve is proposed to evaluate organism exposure to pesticides and organism mortality.

\section{Models gauging pest control interventions inside buildings}

Quantitative threshold to act in relation to pest control are different when pests are inside buildings as there are implications for human health, which cannot easily be measured in terms of economic impact. Ideally, the aim is full pest eradication but since realistically this is unachievable, epidemiology and public health focuses on the threshold between persistence and extinction, gauging it in terms of the impact of chemical treatments in pest populations, through the combination of field experiments with statistical models. Epidemiology models tend to gauge interventions in terms of population decay and health improvements whereas public health studies are more focused on population decay and environmental building conditions.

Two different approaches to interventions are normally present in this case: The fully chemical approach, which consist in using pesticides to control pests, and the integrated management approach which combines building maintenance with sanitation and low toxicity interventions (e.g. baits).

\section{Models of fully chemical approach}

By far the dominant approach is the use of chemicals, with insecticides targeting insects such as mosquitoes and cockroaches, and rodenticides targeting rodents such as mice, rats and squirrels. These pesticides are typically applied with the following methods: spraying for flying insects, surface treatment for crawling insects, fumigation for pests that live inside materials, and baiting for rodents (MacFarlane et al., 2007).

An examples of modelling the fully chemical approach can be found in Rivault and Cloarec (1995) who present a study on the impact of using regular insecticide spray against cockroaches in council flats in France, measuring impact in terms of seeing / counting dead bodies using multivariate principle component analysis to explain cockroach density as function of environmental conditions (mainly related to residents and flat characteristics).

Kass et al. (2009) compare interventions using chemicals with integrated pest management to eradicate cockroaches and mice in urban houses, measuring the impact of each intervention in terms of pest population decay (through sighting and trap counts) and urinary mouse protein and cockroach allergens in dust samples. Linear regression, Poisson regression and logistic regression were used in data analysis. They observed that the apartments in which integrated pest management was used presented significantly lower amounts of cockroaches and sustained this lower counts for a long time period.

\section{Models of Integrated pest management (IPM)}

The increasing concern about the impact of pesticides on building occupants and a broad overview of knowledge about their impact on human health is presented in Kim et al. (2017). Effects range from short term irritation of skin and eyes, headache and nausea to life-threatening and long-term conditions such as asthma, diabetes and cancer. As a result, Integrated Pest Management (IPM) strategies, which are approaches that aim at pest control while at the same time reducing the use of pesticides (Valenti et al., 2018) are gradually being adopted in the built environment. Typically, IPM strategies follow a systematic process that involves prevention, monitoring, pest identification and pest management, and employing pesticides only as last resort (Kalmar et al., 2014).

Rabito et al. (2017) used regression analysis to model the correlation of a reduction in cockroach populations in low income housing with reduction in asthma symptoms in children. The study comes from an epidemiology perspective and the hypothesis is that people are prone to adopt an integrated pest management approach when preventive actions and low cost interventions clearly connect the presence of pests with ill health.

More complex studies are presented by Stejskal 2002 and Rees (2003). Stejskal (2002) makes a distinction between the impact of interventions in local and meta-population of cockroaches. 'The basis of the meta-population theory is that a set of small unstable populations can create one stable large meta-population'. As a result, the traditional pest management approach of eradicating specific sites is insufficient to control meta-populations, meaning pesticides treatment also needs to be applied to 'all suitable refuges that can be recolonized'. The theory suggest pest distribution inside buildings is uneven and that 'the density-dependent allocation of pesticide treatment create partial refuge for pests'. Thus any analysis on the impact of interventions should consider checking the effects on local and meta-population, especially considering the fragmentation of the built environment and the difficulty in accessing all potential infested sites ( ibid.). The persistence of a meta-population changes the threshold control point: 'Meta-population persistence requires that, for a given extinction rate, the colonisation rate exceeds a threshold value, and that for a given colonisation rate the extinction rate is smaller than the threshold value' (Hansel, 1991 in Stejskal, 2002). From this study, one can infer that models of spatial pest distribution inside buildings could be an interesting step further in the study and implementation of IPM in the built environment.

This idea is pursued by Rees (2003) who presents a study in which insect traps are used to monitor the presence, source of infestation and spatial distribution of pests in commercial grain storages. Contour maps, using a geographical mapping program, were produced to indicate the frequency distribution of moths in industrial plants in relation to their internal layout (i.e. position of packing machines, product storage bins, etc.) The findings were important in the planning of targeted control measures, especially in relation to guaranteeing treatment could reach breeding and harbourage areas.

Another interesting study is presented by Valenti et al. (2018) who propose a non-chemical approach to pest control by exploring the use of fan heaters to increase 
inside air temperatures on flour mills, controlling pest infestation through the combined effect of killing by dehydration and altering reproductive patterns. They use thermal modelling and CFD to assess temperatures and heat distribution, connecting the indoor environment with the building envelope in terms of their potential in maximising building response to a non-chemical treatment.

These three studies suggest that pest distribution inside buildings could be seen as analogous to pest distribution on the outside, where the inside is actually a microcosms of the urban habitat, with the last work proposing nonchemical interventions can also be trialled indoors.

\section{Pest proofing the building envelope - No models}

Whereas the approach to pest management on the inside of buildings always considers some kind of chemical treatment, pest control at the level of the building envelope is mainly passive. Design guidelines (Geiger and Cox, 2012; Simons 2005; UK Chartered Institute of Environmental Health, 2018), recommend ensuring that immediate surroundings of buildings are not pest-friendly ecosystems, and that buildings and their conditions are unattractive to pests. Their focus is mainly on ensuring that each common construction assemblage (e.g. foundations, slabs, roofs, etc.) is designed considering:

- Maximum gap sizes to exclude several different types of pests, with specific recommendations for sealants (e.g. use stainless steel meshes in soffit vents, use stainless steel wool and fire block foam, etc.);

- The selection of pest resistant materials, which not only prevent them from entering the building but also deny them harbourage when they reach the inside (e.g. avoid spray foams as they are favourites for rodents, prefer pressure treated wood, use stainless steel meshes, etc.);

- The possibility of being inspected from time to time by inhabitants and professionals to early detect pest incidence (e.g. provide access to foundations, false ceilings, wall voids etc.);

- To avoid moisture conditions inside building materials to prevent pests to flourish (e.g. proper guttering, ventilation of cavity spaces, vapour barriers, etc.);

- Minimise spaces within construction assemblages which can be used for pest harbourage (e.g. false ceilings, wall cavities, false bottoms under cabinets, etc.);

- Eliminate potential building materials that can be used to provide shelter (e.g. rigid foam insulation used for termites to burrow);

- Specific slabs and foundation design in relation to expansion joints (e.g. use of monolithic concrete pours, termite resistant meshes, etc.)
Despite being potentially costly, many of these guidelines clearly pose conflicts with other design goals or compliance codes (Geiger and Cox, 2012), as many places for sheltering pests are actually created by modern methods of construction and building envelope improvements for energy efficient purposes (e.g. wall cavities, pipe insulation, etc.). Besides that, pests actually like similar habitat conditions as human beings and therefore will benefit and flourish in buildings where humans feel comfortable.

\section{Transferability to the built environment, synthesis and discussion}

From recommendations of pest proofing the building envelope, it is possible to see that a pest simulator for the built environment would need to include outdoor models as well as indoor models because succeeding in intervening and controlling the indoor environment are dependent on how pest friendly the outdoor environment is. Thus, when examining each of the aforementioned examples in terms of what is transferable to the built environment it is apparent that a pest simulator would need at least an outdoor environment model, comprehensive enough to describe and predict pests presence, and an indoor environment model, to enable interventions and IPM scenarios to be simulated and assessed when specific epidemiology or public health thresholds are reached. This situation calls for integrated pest models posing questions in relation to how the building envelope is to be taken in consideration in each of these models. Could it be treated analogously to heat transfer models and act as a filter? As the building shell is not only a barrier but also a source of food and harbourage for some pests, how could these be taken into account if the building is considered only as a filter?

Another issue to be discussed is also what kind of mathematical models would be appropriate to describe and predict pests in the outdoor environment and simulate results of interventions and control in the inside when the literature is actually inconclusive in relation to these? From Figure 2, it can be seen that similarities and differences in model characteristics are predominantly related to modelling purposes rather than on the type of mathematical models used. Descriptive models differ among themselves depending on model input data whereas predictive models and models gauging effects of chemical interventions differ according to the various characteristics of each type of intervention, regardless of them happening on the indoor or outdoor environment. Analogous to the latter, integrated pest management models differ based on mechanisms applied to pest population or meta-population control. Decisions in relation to which mathematical models to use are multifactorial and open to future research. 


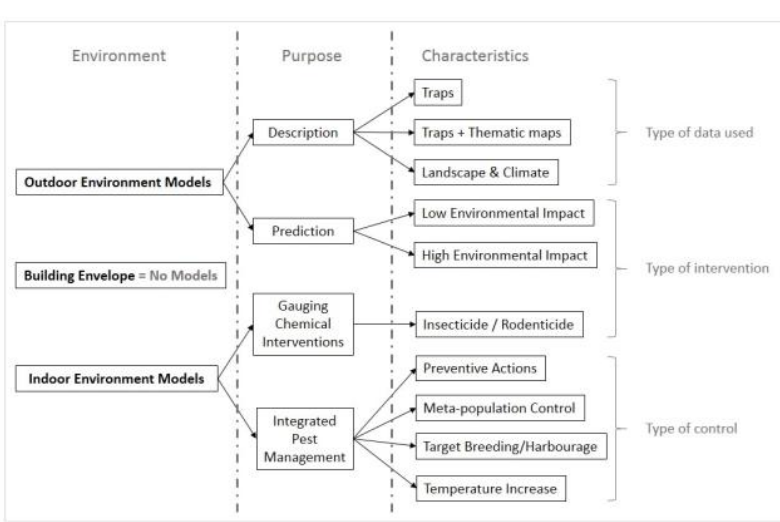

Figure 2: Literature review synthesis

\section{Conclusions and future work}

Despite being standard practice in agriculture and public health, IPM has been slower to become accepted in facility management (Geiger and Cox, 2012) because it is costly and requires special training. By examining recommendations of pest proofing strategies for building envelopes and integrating them with a synthesis of the current state of the art in pest modelling in other domains, the authors expect to open a whole new area to be explored in building performance simulation.

A high level outline of the features of a simulator to assess pest vulnerability in the built environment would initially include the following:

- Making use of outdoor model representations (spatial and georeferenced) as well as comprehensive model data sets, allowing constant updating in terms of pest presence (e.g. from trap data, sighting, etc.) correlated with additions of different environmental and anthropogenic factors which affect them (e.g. microclimate, fine grained land use, socio-economic distribution, different levels of sanitation, etc.). Connect these models with models which gauge different levels of interventions starting from the gradual implementation of passive measures followed by low environmental impact measures (mechanical interventions, and biological methods) up to the use of chemicals.

- Produce indoor models with spatial representations of building layout and room usage to accommodate the uneven distribution of pests inside buildings which, as in outdoor models, accept to be correlated with different factors related to pest presence such as: layout (as analogous to land use), temperature and humidity (as analogous to climate), cleanliness, presence or absence of food, etc. (as analogous to other anthropogenic data) so that interventions to be simulated can be assessed in terms of prevention, control and impact of chemical usage.

Each of these models would need to be pest specific raising a series of question for future work in relation to how the building envelope should be taken into account and consequently what mathematical models should be used to address presence, probability, prevention, treatment and control depending on pest preference for specific types of food and harbourage (which can happen indoors and/or on the building envelope itself) as well as size and ability to penetrate the building shell.

Besides that, whereas thresholds for indoor models can potentially be directly transferred from epidemiology and public health, the same is not true for the outdoor environment in which known thresholds come primarily from agriculture (EIL and ET). Thus, another important question for future work that this study unfolds is what would be an acceptable threshold for intervention in an outdoor urban environment for each different type of pest so a balance between outdoor and indoor interventions can also be simulated.

\section{References}

Akman, O., T. Comar and D. Hrozencik, 2015. On impulsive integrated pest management models with stochastic effects. Frontiers in Neuroscience, 9, article 119

Bonnefoy, X., Kamen, H., Sweeney, K. 2008 - Public health significance of urban pests. World Health Organisation (WHO) Regional Office for Europe. Copenhagen, Denmark.

Caffarra, A., M. Rinaldi, E. Eccel, V. Rossi and I. Pertot, 2012. Modelling the impact of climate change on the interaction between grapevine and its pest and pathogens: European grapevine moth and powdery mildew. Agriculture, Ecosystems and Environment, 148, 89-101.

Chartered Institute of Environmental Health, 2018. Pest minimisation: best practice for the construction industry. Chartered Institute of Environmental Health, London, UK.

del Águila, I., J. Cañadas and S. Túnes, 2015. Decision making models embedded into a web-based tool for assessing pest infestation risk. Biosystems Engineering, 133, 102-115

Diggle, P. J., Moraga, P., Rowlingsin, B., Taylor, B. M. 2013. Spatial and spatio-temporal Log-Gaussian Cox Processes: Extending the geostatistical paradigm. Statistical Science 28 (4):542-563.

Donatelli, M., R. Magarey, S. Bregaglio, L. Willocquet, J. Wish and S. Savary, 2017. Modelling the impacts of pests and diseases on agricultural systems. Agricultural Systems, 155, 213-224

FAO/IAEA Programme 2006. Designing and implementing a geographical information system. A guide for managers of area-wide pest management programmes. Sponsored by the joint FAO/IAEA Programme of Nuclear Techniques in Food and Agriculture. IAEA Vienna

Geiger, C. A., Cox, C. 2012. Pest prevention by design: Authoritative guidelines for designing pests out of structures. San Francisco Department of Environment, USA. 
Georgescu, P. and H. Zhang, 2010. An impulsively controlled predator-pest model with disease in the pest. Nonlinear Analysis: Real World Applications, $11,270-287$

Gilioli, G., S. Pasquali and E. Marchesini, 2016. A modelling framework for pest population dynamics and management: an application to the grape berry moth. Ecological Modelling, 320, 348-357

Kalmar, E., S. Ivey, A. Bradman, V. Leonard and A. Alkon, 2014. Implementing an integrated pest management (IPM) program in child care centres: a qualitative study. Early Childhood Research Quarterly, 29, 245-254

Kass, D., W. McKelvey, E. Carlton, M. Hernandez, G. Chew, S. Nagle, R. Garfinkel, B. Clarke, J. Tiven, C. Espino and D. Evans, 2009. Effectiveness of an Integrated Pest Management intervention in controlling cockroaches, mice, and allergens in New York City public housing. Environmental Health Perspectives, 117, 1219-1225

Kim, K., E. Kabir and S. Ara Jahan, 2017. Exposure to pesticides and the associated human health effects. Science of the Total Environment, 575, 525-535

Luo, L., D. Ashworth, J. Šimunek, R. Xuan and S. Yates, 2013. Assessment of methods for methyl iodide emission reduction and pest control using a simulation model. Atmospheric Environment, 66, 3340

MacFarlane, E. G. Benke, D. Goddard and M. Sim, 2007. Urban pest control operators in Australia. Occupational and Environmental Medicine, 64 (6), 422-42

Mamedov, A. and S. Udalov, 2002. A computer tool to develop individual-based models for simulation of population interactions. Ecological Modelling, 147, 53-68

Murray, J., D. Berman and R. van Klinken, 2014. Predictive modelling to aid the regional-scale management of a vertebrate pest. Biological Invasions, 16, 2403-2425

Patton, M. O. 2002. Qualitative Research and Evaluation Methods. Thousand Oaks, CA: Sage.

Petrovskii, S., N, Petrovskaya and D. Bearup, 2014. Multiscale approach to pest insect monitoring: random walks, pattern formation, synchronization, and networks. Physics of Life Reviews, 11, 467-525

Rabito, F., J. Carlson, H. He, D. Werthemann and C. Schal, 2017. A single intervention for cockroach control reduces cockroach exposure and asthma morbidity in children. 140 (2), 565-570

Rees, D., 2003. Monitoring insect pests within buildings using traps: case studies of the use of traps to monitor activity, spatial distribution and efficacy of pest control. AICCM Bulletin, 28 (1), 44-49.
Rentokil, 2015. The Rentokil Report 2015 - Insights from pest control markets across the globe. Camberley: Rentokil.

Rivault, C. and A. Cloarec, 1995. Limits of insecticide cockroach control in council flats in France. Journal of Environmental Management, 45, 379-39

Sacchi, R., Gentilli, A., Pilon, N., Bernini, F. 2008. GIS modelling the distribution of Rattus Norvegicus in urban areas using non-toxic attractive baits. Hystrix, the Italian journal of mammalogy 19 (1).

Shirley, M., S. Pushton, A. Young and G. Port, 2001. Simulating the long-term dynamics of slug populations: a process-based modelling approach for pest control. Journal of Applied Ecology, 38, 401-411

Simons, S. 2005. Pest prevention construction guidelines and practices. Journal of School Business Management 70(4):10-16.

Stejskal, V. 2002. Metapopulation concept and the persistence of urban pests in buildings. Proceedings of the $4^{\text {th }}$ Interantional Conference on Urban Pests. In Jones, S. C., Zhai, J., Wm H. Robinsons Editors.

Sutherst, R., 2014. Pest species distribution modelling: origins and lessons from history. Biological Invasions, 16, 239-256

Tamayo-Uria, I., Mateu, J., Diggle, P. J. 2014. Modelling of the spatio-temporal distribution of rat sightings in an urban environment. Spatial Staistics 9: 192-206.

Tang, S., G. Tang and R. Cheke, 2010. Optimum timing for integrated pest management: modelling rates of pesticide application and natural enemy releases. Journal of Theoretical Biology, 264, 623-638

Traweger, D. and Slotta- Bachmayr, L. 2005. Introducing GIS-modelling into the management of a brown rat (Rattus norvegicus Berk.) (Mamm. Rodentia Muridae) population in an urban habitat. Journal of Pest Science 78:17-24.

Valenti, F., S Carmela Porto, N. Tomasello and C. Arcidiacono, 2018. Enhancing heat treatment efficacy for insect pest control: a case study of a CFD application to improve the design and structure of a flour mill. MDPI Buildings, 8 (48), doi:10.3390/buildings8040048.

Wang, S., J. Dou and L. Lu, 2011. A pest control model with periodic coefficients and impulses. Procedia Environmental Sciences, 8, 506-513

Zhang, W. and S. Swinton, 2009. Incorporating natural enemies in an economic threshold for dynamically optimal pest management. Ecological Modelling, 220, 1315-1324. 